\title{
Volume Scattering and Echo Integration in Fisheries Acoustics Revisited
}

\section{Masahiko Furusawa}

Tokyo University of Marine Science and Technology, Japan, mfrsw@outlook.jp

Follow this and additional works at: https://jmstt.ntou.edu.tw/journal

Part of the Fresh Water Studies Commons, Marine Biology Commons, Ocean Engineering Commons, Oceanography Commons, and the Other Oceanography and Atmospheric Sciences and Meteorology Commons

\section{Recommended Citation}

Furusawa, Masahiko (2021) "Volume Scattering and Echo Integration in Fisheries Acoustics Revisited," Journal of Marine Science and Technology. Vol. 29: Iss. 2, Article 1.

DOI: 10.51400/2709-6998.1074

Available at: https://jmstt.ntou.edu.tw/journal/vol29/iss2/1

This Research Article is brought to you for free and open access by Journal of Marine Science and Technology. It has been accepted for inclusion in Journal of Marine Science and Technology by an authorized editor of Journal of Marine Science and Technology. 


\title{
Volume Scattering and Echo Integration in Fisheries Acoustics Revisited
}

\author{
Masahiko Furusawa
}

Tokyo University of Marine Science and Technology, Japan

\begin{abstract}
Echo integration is the most important method in fisheries acoustics. The basis of the method is volume scattering theory, and the theory assumes that many fish are randomly and homogeneously distributed broader than a beam spread. In actual acoustic surveys, however, we encounter various distributions for which these assumptions do not hold. It is the echo integration method that hypothetically fulfills these assumptions. In this paper, we review the volume scattering theory and the echo integration method historically and theoretically and confirm their bases. We then point out some misunderstandings affected by historical constraints such as the persistence of time integration. We also introduce and discuss some useful variants of the echo integration, especially calibration by standard-sphere echo integration.
\end{abstract}

Keywords: Volume scattering, Echo integration, Standard-sphere calibration

\section{Introduction}

$\mathrm{E}$ cho integration (EI) is the most important method for acoustic fish abundance estimation. There are, however, some different interpretations of the EI, and then their scrutinization is needed. Some interpretations are affected by historical constraints such as the persistence of time integration, and they sometimes decrease the power of the EI. The basis of the EI is volume scattering theory (VST). It is important to understand clearly the relation between the EI and VST when applying them. In particular, applications of the EI to rather new areas such as the EI in standard-sphere calibration need sufficient understanding of the VST and EI. In this paper, we first review the VST and EI historically and theoretically to confirm their fundamentals, and then discuss some applications.
Since sometimes there appears essence in primitive or earlier phases, in Sec.II we first review the history of the VST and EI. We consider the principle and constraint of the VST in Sec.III. Section IV elucidates the principle and applications of the EI. We examine, in Sec.V, the characteristics of the VST and EI using a statistical model. In Sec.VI we explain the basic processing in quantitative or scientific echosounders. Finally, in Sec.VII, we introduce and discuss some variants of the EI, especially the EI of standard-sphere echoes.

\section{Historical consideration}

The volume scattering or reverberation theory (VST) appeared as early as in 1948 in [4]. They established the VST for underwater objects such as a deep scattering layer and also the surface scattering theory from the sea surface and bottom, and measured their approximate scattering strengths. They read average echo levels from the photographs of oscilloscope displays ("scope reading") and 
demonstrated the scattering levels against the range.

Saneyoshi and Nakamura (1952) [22] conducted experiments in air for fish school models which were the arrays of randomly suspended rubber balls. They confirmed the basic characteristics of the VST such that the square root of the number of balls was proportional to the average echo voltage. Their method was also the scope reading and averaging for pings.

Truskanov and Sherbino (1964) [30] (provided as Appendix II of [26]) confirmed that the square root of fish distribution density was proportional to average echo voltage, and then applied the relationship to the abundance estimation of cod and herring. Their method was again the scope reading and ping averaging.

In the earlier papers cited above, from the historical constraint, the scope reading and ping averaging method had been used to observe the distribution density of fish against range. The method did not perform the echo integration over the range or time which is commonly used in present-day echo processing, and could accomplish density measurements directly following the VST.

Since such a method was laborious, timeconsuming, and did not give accurate and precise results, a method of echo integration over time or range was introduced. Earlier echo integration methods, however, were the analogue integration of echo voltage, also from the historical constraint. Dragesund and Olsen (1965) [3] developed a voltage integrator for two depth layers, and confirming the fact that the results were proportional to the square root of distribution density, they used the integrator for actual fish abundance estimation.

Thorne (1971) [29] also used a voltage integrator and confirmed that the integrated values were proportional to the fish number for single fish echoes, and that the squared results were proportional to the fish number for multiple echoes. In his paper, he predicted that a more sophisticated method, i.e. the echo integration for squared echo voltage should accomplish density measurements both for single and multiple echoes.

In the FAO manual which was edited by Forbes and Nakken (1972) [5], the density measurements both by the VST and the EI method for squared voltage were introduced, and the theory and method were similar to the present form. But the integrator was analogue and the number of integration layers was not many. Also in the manual, a convenient measure, i.e. the area backscattering strength, appeared. Further, an absolute calibration method using a calibrated hydrophone was introduced instead of the traditional relative calibration method comparing acoustic estimates with net catches or fish number in net-cages.

In the later manuals by Burcziskii (1982) [1] and Johanesson and Mitson (1983) [11], together with the principle of density measurement according to the VST, multi-layer digital echo integration was introduced. In the early years of the digital EI, since the processing speed and memory capacity were not sufficient, the number of integration layers was small such as ten and the flexibility in echo selection was not high.

Furusawa et al. (1989) [6] devised therefore a method termed the two-step echo integration method: in the first step echoes were integrated in small cells of which size were the maximum range/ $50 \times$ e.g. $0.2 \mathrm{nmi}$, and the results were stored once on for example a floppy disc; in the second step the small cell data were read and given some weights for echo selection and integrated again in large cells.

Until these years the VST but Maclennan (1990) [15], MacLennan and Simmonds (1992) [16], and Simmonds and MacLennan (2005) [24] introduced the echo integrator equation which combined the VST and the EI over time or range, and the result of the equation was not the volume density but the area density.

In recent years, an expression called a power budget equation, which originated from a radar equation and based on power, has been introduced $[2,25]$. Instead of the traditional sonar equation based on intensity [31], the equation amalgamated the VST and EI in its own form [13]. There is, however, some confusion in the theory and applications, particularly in the interpretation of the EI, which we will discuss later.

Since modern quantitative echosounders digitize echo data at high speed on the earlier stage of the receiver and use a large capacity memory, the constraint in processing echoes is greatly alleviated. That is, basic data are obtained for small pixels of which horizontal size is one ping and vertical size is one sampling period (e.g., the depth interval is $5 \mathrm{~cm}$ for a sampling frequency of $15 \mathrm{kHz}$ ), and then we can nearly freely conduct real-time and post processing.

Summarizing the aforementioned history, the echo processing methods have advanced as 1) the scope reading and ping averaging, 2) the analogue echo integration of voltage, 3 ) the analogue echo integration of squared voltage, 4) the multiple-layer digital echo integration, 5) the two-step echo integration, and 6) the pixel echo integration. The theoretical change is summarized as 1) only the VST, 2) the EI of VST results, and 3) the merging of the VST and EI. This paper will claim that the VST and EI are better to be separated [i.e. above 2)]. 


\section{Volume scattering theory}

Sound intensity $I$ is a convenient quantity to express sound waves. The intensity is proportional to the pressure squared $P^{2}$ :

$I=\frac{P^{2}}{\rho c}$

where $\rho$ is the water density and $c$ is the sound speed in water. Since the specific acoustic impedance $\rho c$ is nearly constant, the intensity $I$ and the pressure squared $P^{2}$ may be considered to be equivalent. The pressure is proportional to voltage $E$ and echoes are processed for the voltage or its digitized value. For example, the echo signal voltage is simply expressed as

$E=P M G$

where $M$ is the receive sensitivity of a transducer and $G$ is the receiver gain. Therefore the pressure (squared) and echo voltage (squared) are mainly used in this paper.

We use different acoustic terminology and symbols as shown in the appendix table (Table A [10]) from those recommended by the International Council for the Exploration of the Sea (ICES) [17], but they can be easily transformed by referencing the table.

It is convenient to develop a sonar equation for multiple echoes, that is equivalent to the volume scattering theory (VST), from a sonar equation for single fish echoes. Referencing Fig.1, the single echo pressure squared $P_{S}{ }^{2}$ is expressed as

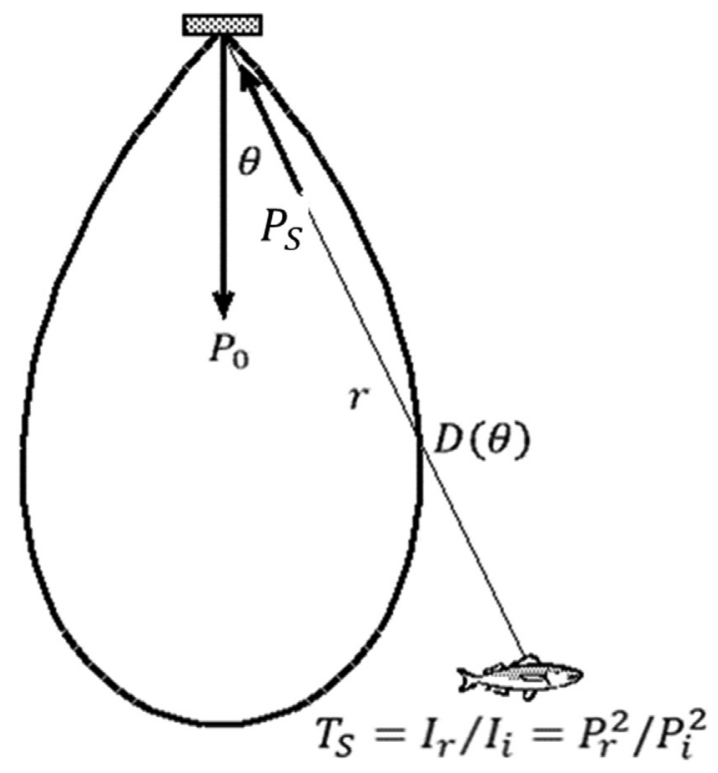

Fig. 1. Derivation of single fish echo equation.

$$
\begin{aligned}
P_{S}^{2} & =P_{0}^{2} D(\theta, \phi)^{2} \frac{1}{r^{2} 10^{0.1 \alpha r}} T_{S} \frac{1}{r^{2} 10^{0.1 \alpha r}} D(\theta, \phi)^{2} \\
& =P_{0}^{2} \frac{1}{r^{4} 10^{0.2 \alpha r}} D(\theta, \phi)^{4} T_{S}
\end{aligned}
$$

where $P_{0}$ is the source pressure, $D(\theta, \phi)$ is the pressure directivity function for a fish at a direction $\theta$ and $\phi, r$ is the range from the transducer to the fish, $\alpha$ is the absorption coefficient in $\mathrm{dB} / \mathrm{m}, T_{S}$ is the linear value of the target strength (abbreviated as TS; see Table A). The first equation arranges factors in the order of the transmission, attenuation in the outgoing path, reflection, attenuation in the returning path, and receiving.

The macroscopic theory of the VST $[4,31]$ is easily derived from the sonar equation for the single echo, Eq. (3). If we observe echoes of fish in an incremental solid angle $d \Omega$ at a range $r$, then fish of which echoes are synthesized there should be in the range $c \tau / 2$, where $\tau$ is the pulse width (Fig.2). Thus the fish are to be in the incremental volume:

$d V=r^{2} d \Omega \frac{c \tau}{2}$

If fish distribution density is $n\left[1 / \mathrm{m}^{3}\right]$, then the contribution in scattering from this volume becomes $n d V$ times $T_{S}$. Replacing $T_{S}$ in Eq. (3) by this amount, we have

$d P_{M}^{2}=P_{0}^{2} \frac{1}{r^{4} 10^{0.2 \alpha r}} D^{4}(\theta, \phi) T_{S} n d V$

Integrating this over a hemi-sphere gives

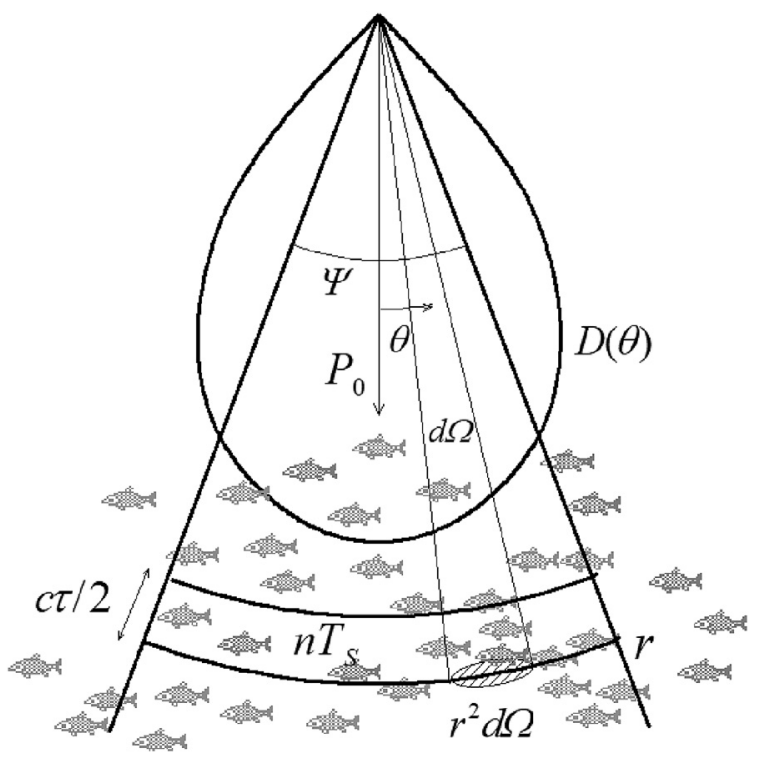

Fig. 2. Derivation of multiple echo equation. 


$$
\begin{aligned}
& P_{M}^{2}=\int_{\pi} d P_{M}^{2}=P_{0}^{2} \frac{1}{r^{2} 10^{0.2 \alpha r}} \frac{c \tau}{2} \Psi S_{V} \\
& \Psi=\int_{\pi} D^{4}(\theta, \phi) d \Omega \\
& S_{V}=n T_{S}
\end{aligned}
$$

where $\Psi$ is the equivalent beam angle, $S_{V}$ is the volume backscattering strength (linear value; abbreviation is SV; see Table A). This expression exhibits the composition of echoes from fish in the scattering volume:

$V_{s}=\frac{c \tau}{2} \Psi r^{2}$

From Eq. (6) the expression for SV measurements becomes

$S_{V}=\frac{P_{M}^{2} r^{2} 10^{0.2 \alpha r}}{P_{0}^{2}(c \tau / 2) \Psi}$

The abovementioned macroscopic theory can be applied in most cases, but when we must consider more strictly and precisely, we need microscopic theory which includes individual echo waveforms (e.g. [7]). The composed echo amplitude of many single echoes, of which amplitudes are shown by Eq. (3), is expressed as

$P_{M}^{2}=\sum_{k=1}^{N} P_{S, k}^{2}+\sum_{k=1}^{N} \sum_{\substack{n=1 \\ k \neq m}}^{N} P_{S, k} P_{S, m} \cos \left(\gamma_{k}-\gamma_{m}\right)$

where $N$ is the number of fish that contribute scattering at some time corresponding to a range $r$ shown in Fig.2, $\gamma$ is the initial phase angle of each fish echo, and $k$ and $m$ are the indexes of fish. The average value of the second term (interfering component) is zero because $\gamma$ can be assumed to be random, but the number of the interfering echoes $N(N-1)$ is large to give a considerably large variance as shown later. Thus with some averaging processing we make the equation to be expressed only by the first term (basic component).

Analysis of the basic component gives the following expressions for the range and pulse width in Eq. (6) [7]:

$$
\begin{aligned}
& r \rightarrow r-\delta r \cong r-\frac{c \tau_{0}}{4} \\
& \tau=\int_{0}^{\tau_{m}} w^{2}(t) d t
\end{aligned}
$$

where $\delta r$ is the range correction determined to make a near range error small and approximately shown by $c \tau_{0} / 4, \tau_{0}$ is the transmit pulse width, $\tau$ is the equivalent pulse width, $w(t)$ is the waveform function expressing the envelope of the single echo, and $\tau_{m}$ is its duration time (see Fig.3a). Caution should be exercised in that the definition of the equivalent pulse width Eq. (13) is the result of superposition of the multiple echoes in volume scattering as shown in Fig.3d (i.e. at an observation instant many points of the wave function squared are composed) and not the result of a priori time integration processing.

In the development of the VST given above, we find that the theory needs the four assumptions: 1 ) many fish are distributed within the beam; 2) they are distributed randomly and homogeneously; 3) the school size is larger than the beam spread; 4) some averaging process is necessary to realize high precision. These assumptions except 4 ) are fulfilled only near the central part of a large school or for a broadly distributed layer of fish, and in many cases these assumptions do not hold. It is the echo integration that hypothetically realizes these assumptions.

\section{Echo integration}

We apply ensemble average to Eq. (11) over ping $j$ and have

$$
\begin{aligned}
<P_{M}^{2}> & =\frac{1}{J} \sum_{j=1}^{J}\left[\sum_{k=1}^{N_{j}} P_{S, k}^{2}+\sum_{k=1}^{N_{j}} \sum_{\substack{n=1 \\
k \neq m}}^{N_{j}} P_{S, k} P_{S, m} \cos \left(\gamma_{k}-\gamma_{m}\right)\right] \\
& \cong \frac{1}{J} \sum_{j=1}^{J} \sum_{k=1}^{N_{j}} P_{S, k}^{2}=\frac{1}{J} \sum_{k=1}^{\Sigma N_{j}} P_{S, k}^{2}
\end{aligned}
$$

The last expression can be schematically modeled in Fig.4: various echoes, such as single echoes and multiple echoes, and even empty space, are superimposed and then we have a hypothetical large school which realizes the abovementioned assumptions 1) to 3) shown in Sec. III. The averaging makes it possible to ignore the second term in the brackets that is the interference component, and realizes the assumption 4 ).

This is the definition of the echo integration (EI), and is a little bit different from the definition that gives weight to the time integration which is affected by the historical constraint. The EI is the method to alleviate the limitation of the volume scattering theory (VST) by introducing the ping averaging and to extend the applicability of the VST. 


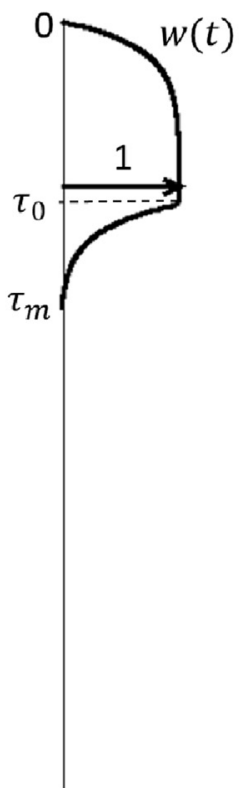

a) Wave function
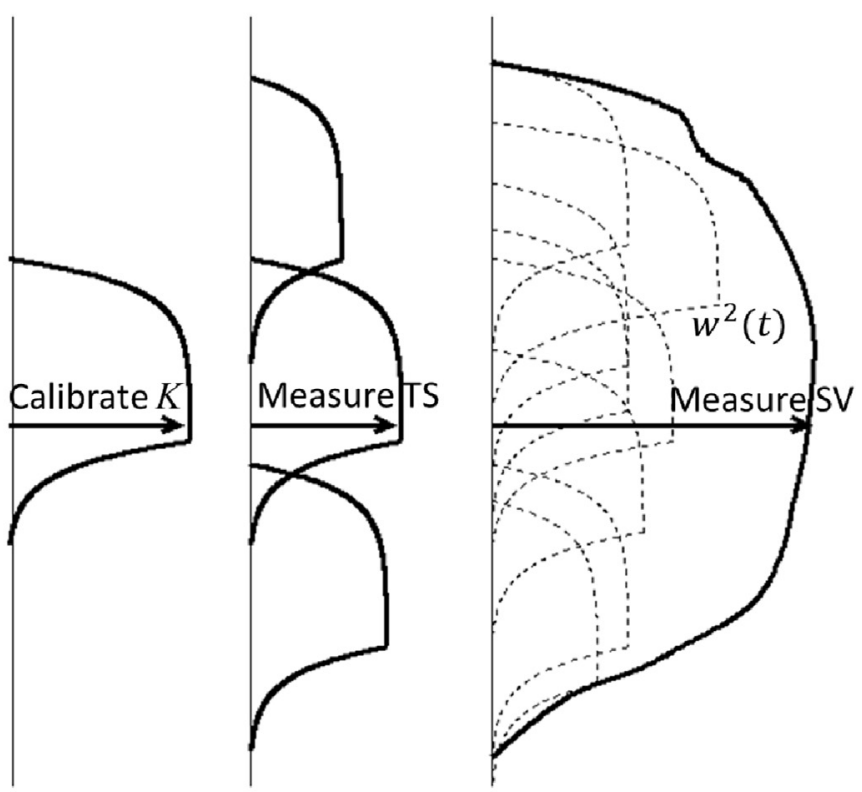

b) Sphere echo d) Multiple echo

Fig. 3. Wave function and measurement points.

The ping averaging of scope readings as reviewed in Sec.II meets this definition. We here remember the sentence "signal averaging or integration to obtain fish densities" in the earlier manual [5]. A more proper name for the echo integration method thus would be the echo averaging method. Conducting a proper processing according to the abovementioned definition, the dead zone just above a seabed is considerably alleviated [8].

Owing to the high speed and large capacity memory of present-day computers, we first obtain, according to Eq. (10), non-averaged raw or pixel SV's, $S_{V, i j}$, for each sample range and ping, where $i$ is the range index and $j$ is the ping index (Fig.5). The ping average SV corresponding to Eq. (14) is expressed as

$$
<S_{V}>_{i}=\frac{1}{J} \sum_{j=1}^{J} S_{V, i j}
$$

where $J$ is the number of pings (or integration period).

In our fish echo case, we can apply the ergodicity principle [21] that claims that the ensemble average (ping average) and time average (range average) are equivalent, and then we can increase the number of averaging with the time averaging and get more precise results. The result of this averaging is called the cell or layer average SV and shown as
$<S_{V}>=\frac{1}{I} \sum_{i=1}^{I}\left[\frac{1}{J} \sum_{j=1}^{J} S_{V, i j}\right]$

where $I$ is the number of samples along range (or integration layer width).

From the above discussion it is better to discriminate the SV into the following three types (see Fig.5).

1) Raw (or pixel) SV: value of Eq. (10) processed according to the VST, assuming to be a multiple echo.

2) Intrinsic (or true) SV: SV actually fulfilling the volume scattering conditions.

3) Average SV: averaged raw SV's.

The area backscattering strength (SA) is a practical and useful EI result for fish abundance estimation. The definition is (see Table A)

$S_{A}=\int_{r_{1}}^{r_{2}} S_{V} d r=n_{A}<T_{S}>$

where $r_{1}$ to $r_{2}$ shows an integration range which defines the SA layer, $n_{A}$ is the area density $\left[1 / \mathrm{m}^{2}\right]$, and $\left\langle T_{S}\right\rangle$ is the average TS. The layer SA is 

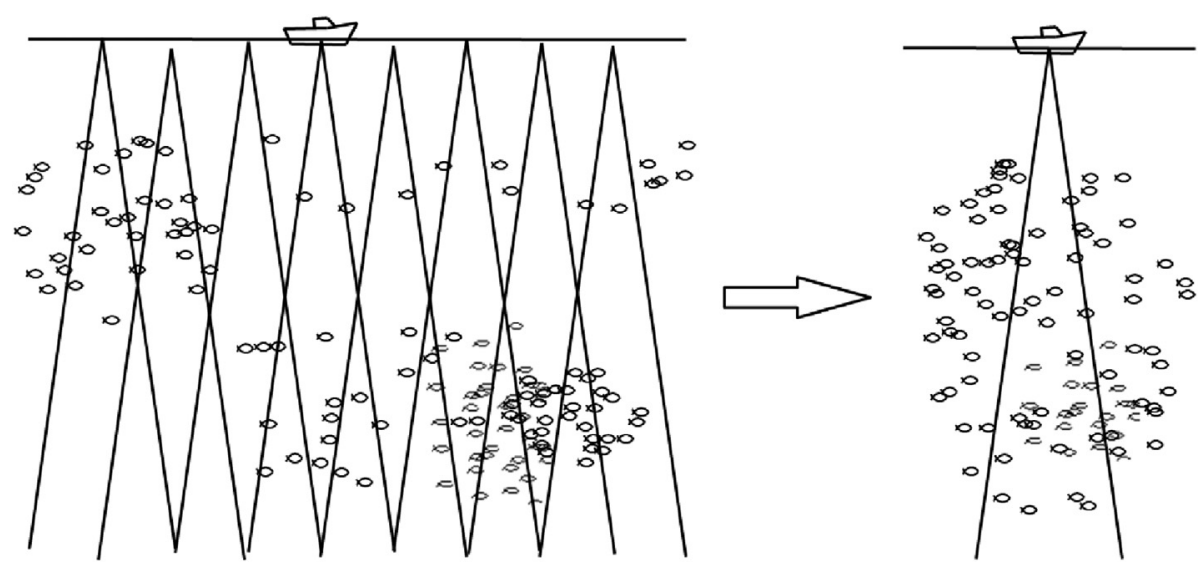

Fig. 4. Schematic concept of echo integration. Echoes for each ping (left) are accumulated to give a hypothetical large school echo (right).

$S_{A}=\Delta r \sum_{i=1}^{I}\left[\frac{1}{J} \sum_{j=1}^{J} S_{V, i j}\right]$

where $\Delta r$ is the range step corresponding to a sampling interval (Fig.5). For present-day post processing, the most useful SA definition is the region SA:

$S_{A}=\Delta r \sum_{i=1}^{I}\left[\frac{1}{J} \sum_{j=1}^{J} m_{i j} S_{V, i j}\right]$

where $m_{i j}$ are the masks given by echo selection processing (see Fig.6) and 1 for selected raw SV's and 0 for ones to be rejected.

\section{Consideration by a stochastic model}

Here we consider the characteristics of the VST and EI more in detail using an inhomogeneous
filtered-Poisson-process model [19]. The normalized variance (squared coefficient of variation) of the echo integrated outputs in terms of this model is, with some replacement of variables (see Appendix II),

$\frac{\sigma_{I}^{2}}{\mu_{I}^{2}}=\frac{1}{J}\left[\frac{\mathrm{E}\left\{F^{4}\right\}}{\left(\mathrm{E}\left\{F^{2}\right\}\right)^{2}} \frac{3}{n \Psi\left(r_{2}^{3}-r_{1}^{3}\right)}+\frac{3 c \tau\left(r_{2}^{5}-r_{1}^{5}\right)}{5\left(r_{2}^{3}-r_{1}^{3}\right)^{2}}\right]$

where $\mu_{I}^{2}$ is the squared average of the EI outputs, $\sigma_{I}^{2}$ is the variance, $J$ is the ping number for averaging, $r_{1}$ to $r_{2}$ is averaging range (integration layer), and $\mathrm{E}\left\{F^{4}\right\} /\left(\mathrm{E}\left\{F^{2}\right\}\right)^{2}$ is the moment ratio of random amplitude factors $F$, and is equal to two assuming the Rayleigh distribution of TS. The first term in the brackets represents the variance caused by the scarcity of fish within the beam, while the second term by the scarcity of independent echoes.

First we observe the general characteristics of the normalized variance as a function of fish density $n$

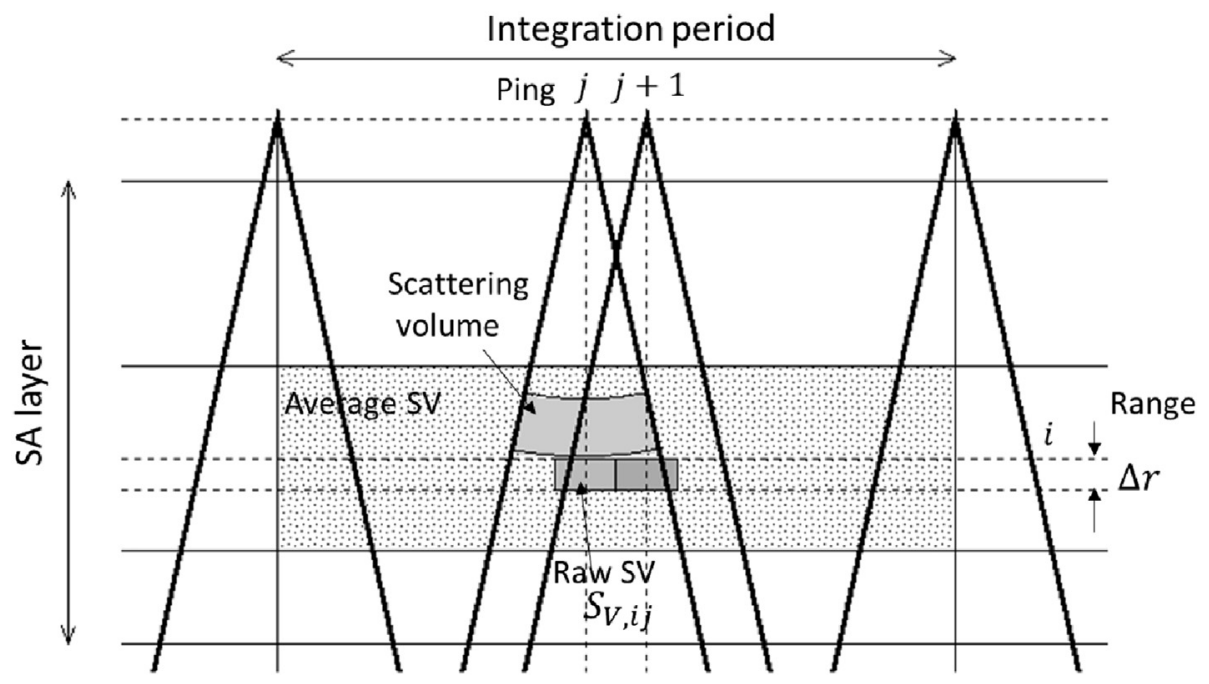

Fig. 5. Pixel volume-backscattering-strengths (SV) are obtained for each ping $j$ and sampled range $i$. They are averaged to give average SV. 


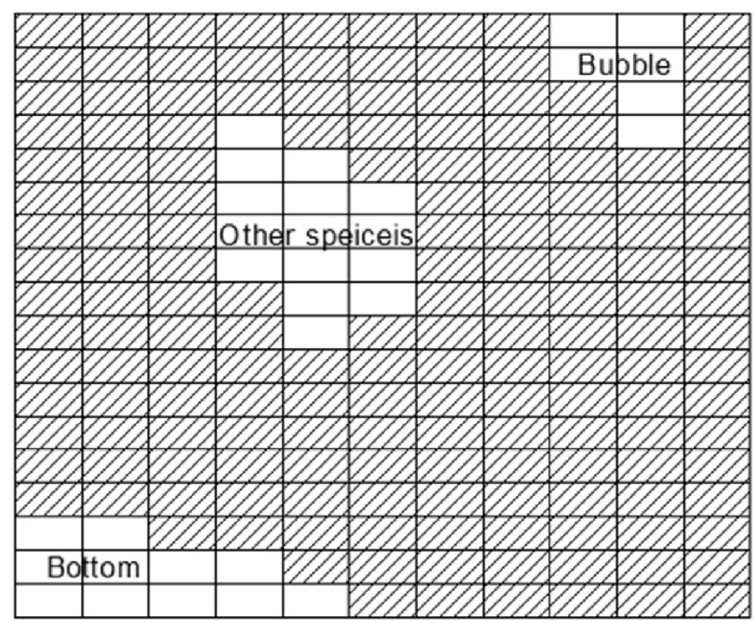

Fig. 6. Schematic concept of region area-scattering-strength (region SA). Shaded pixels are assigned mask values of one while white pixels zero.

(Fig.7). From the need of single echo measurements, we select a small beam width (or $\Psi$ ) and small $\tau$ as shown by the solid line, and this makes the first term of Eq. (20) large and the second term small. Thanks to the effect of the echo integration, however, the variance is small even for considerably sparse distribution of fish.

In the case of the minimum averaging, $r_{2}-r_{1}=$ $c \tau / 2$ and $J=1$, we have Fig. 8 where the variable is the range, the distribution density $n$ are varied as $0.01,0.1$, and $1 / \mathrm{m}^{3}$, the pulse width $\tau=1 \mathrm{~ms}$, and the beam width is $7^{\circ}(\Psi=0.0083 \mathrm{sr})$. The normalized variances are large for small densities and small ranges, but as they become large the curves

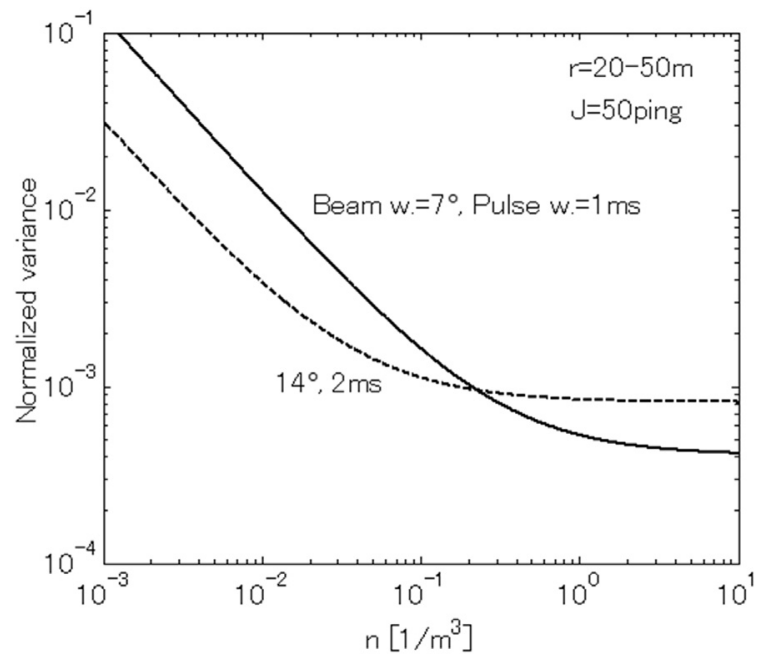

Fig. 7. Normalized variance of echo integration results as a function of distribution density of fish for two beam-width and pulse-width combinations. Integration is done over 50 pings and 20-50 $\mathrm{m}$ range. The equivalent beam angles $\Psi$ are 0.0083 and $0.0330 \mathrm{sr}$ for $7^{\circ}$ and $14^{\circ}$ beams, respectively.

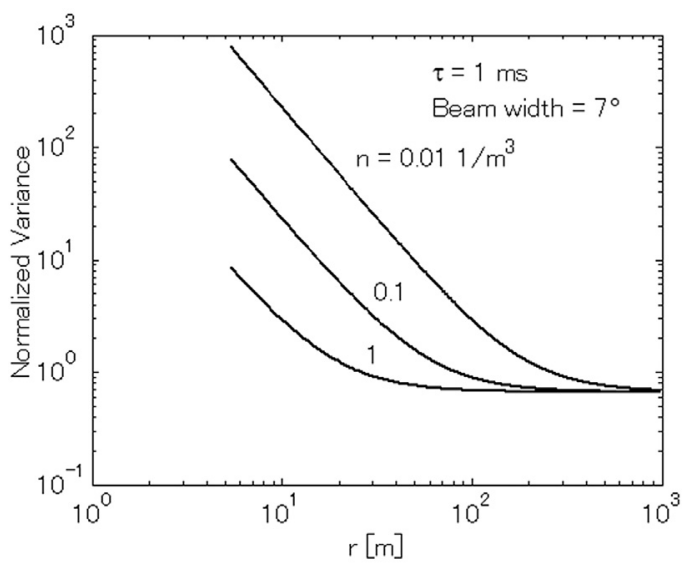

Fig. 8. Normalized variance as a function of range for three densities in the case of no averaging.

asymptotically approach a constant value of $2 / 3$ (see Appendix II) at the region where the VST holds. This minimum value corresponds to the interference component of Eq. (11). If we assume that the asymptotic value starts where the first term becomes $\kappa$ times the second term, then we have the fish number in the scattering volume (see Appendix II).

$n \Psi r_{2}^{2} \frac{c \tau}{2} \cong \frac{3}{\kappa}$

and if we choose the value $\kappa=0.1$ we have a reasonable value of 30 fish for volume scattering.

We examine if the ergodicity holds. When $r_{1}$ and $r_{2}$ separate by a distance larger than $c \tau / 2$, then echo data can be assumed to be approximately independent, and then $I_{r}=\left(r_{2}-r_{1}\right) /(c \tau / 2)$ can be seen as the index of averaging number corresponding to $J$ for the ping averaging. Fig. 9 compares the ping

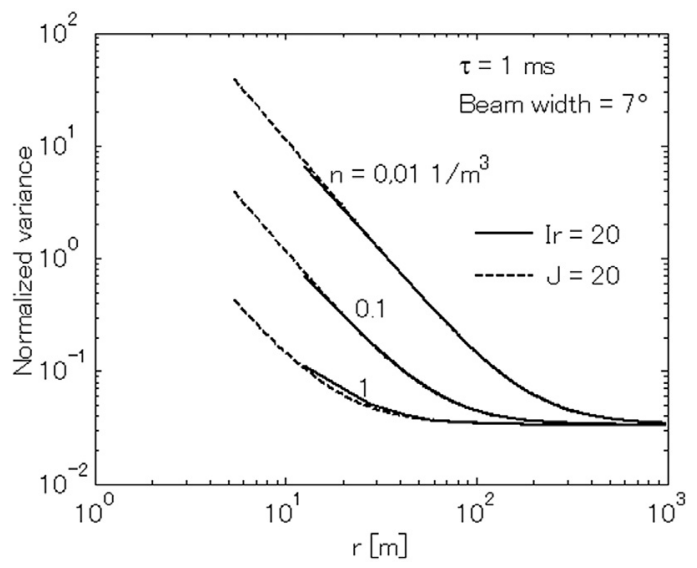

Fig. 9. Comparison of normalized variance for ping integration (dashed lines) and range integration (solid lines) to confirm ergodicity principle. 
averaging $\left(J=20, I_{r}=1\right)$ and range averaging $(J=1$, $\left.I_{r}=20\right)$ and demonstrates almost the same normalized variance to validate the ergodicity. Hence the product number $J I_{r}$ can be seen as a total number of averaging having the same effect as the number of ping averaging.

From this fact, shifting the curves in Fig. 8 by $1 /$ $\left(J I_{r}\right)$, we can see the effect of averaging. The graph shows that even if echoes in the region of small ranges and low densities exhibit a large variance, they become like multiple echoes with a small variance owing to the averaging or integration process. An example: if a vessel speed is $10 \mathrm{kt}$, a pulse repetition period is $1 \mathrm{~s}$, and an integration interval is $0.1 \mathrm{nmi}$, then $J=0.1 / 10 \times 3600 / 1=36$, and if a pulse width is $1 \mathrm{~ms}$ and an integration width is $30 \mathrm{~m}$, then $I_{r}$ is 40 , then we have $J I_{r}=1440$; this averaging number realizes a reasonable measurement for a fish distribution of density $0.01 \mathrm{~m}^{-3}$ at 40 $\mathrm{m}$ depth, because the original normalized variance 15.2 becomes about $15.2 / 1440=0.01$. Thus Fig. 8 can be seen as the universal graph.

\section{Basic processing}

From the above discussion the block diagram of the basic processing of a quantitative (or scientific) echosounder is shown as Fig.10.

From Eqs. (2) and (3) the TS is expressed by the single echo voltage $E_{S}$ :

$$
\begin{aligned}
& \sqrt{T_{S}}=E_{S} \frac{r^{2} 10^{0.1 \alpha r}}{K D^{2}} \\
& K=P_{0} M G
\end{aligned}
$$

The single echo channel (the upper channel of Fig.10) compensates for the range dependent factor by a so-called $40 \log r$ TVG, the directivity $D^{2}$ by, for example, a split-beam method, and the transmitreceive factor $K$ by calibration. Multiple echoes also enter into this channel, but are blocked by the single echo extraction processing.

Multiple echoes are transformed to the voltage signal $E_{M}$ by Eq. (2) and then to raw SV by Eq. (10) in the lower channel:

$$
\begin{gathered}
S_{V}=E_{M}^{2} \frac{r^{2} 10^{0.2 \alpha r}}{K_{M}^{2}} \\
K_{M}^{2}=K^{2}(c \tau / 2) \Psi
\end{gathered}
$$

where $K_{M}{ }^{2}$ is termed the multiple-echo factor and given by the calibration. The range factor is corrected by a so-called $20 \log r$ TVG. Single echoes are similarly processed and the succeeding EI processing makes them the average SV.

\section{Variants}

The abovementioned echo integration (EI) processing is the orthodox one applied to ordinary acoustic surveys of fish abundance. There are some variants of EI that support the surveys. These are, however, just the variants and it is necessary to discriminate them from the orthodox EI. We first briefly introduce the variants reported by the present author's group, and next we discuss the EI of a standard-sphere echo that is one of the present-day important calibration methods.

The EI is fundamentally mean square processing of signals, and therefore can be used for average power measurements. Takao and Furusawa (1995) [27] devised a noise measurement method using echo integrated data, but with inverse TVG processing. The method can provide noise levels comparable with other vessels or instruments.

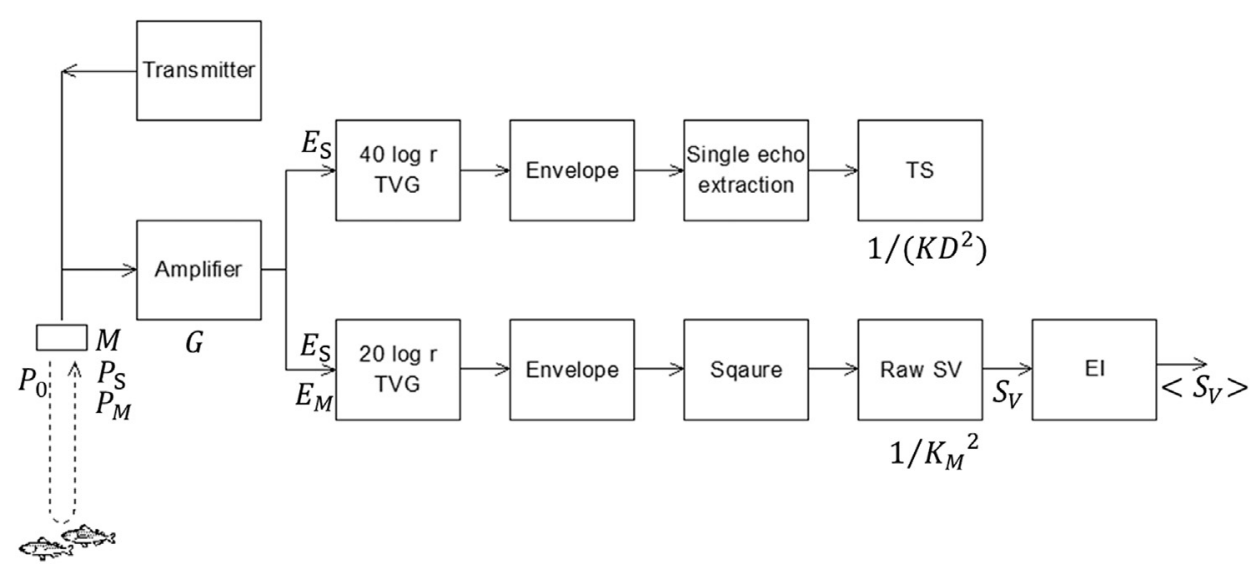

Fig. 10. Basic processing by quantitative echo sounder. The upper channel followed by "40 log $r$ TVG" is for single echoes and the lower channel followed by "20 $\log r$ TVG" is both for single and multiple echoes. 
The scattering from sea bottoms is mainly surface scattering differing from volume scattering. Conducting the EI, however, for a range including bottom echoes gives easily and precisely the surface scattering strength of the bottom [18], which serves to estimate bottom properties.

Scattering measurements of individual schools are important for fisheries and fish species identification. At the edge of a school or for a small school, the equivalent beam angle shown by Eq. (7) becomes too large because of uneven distribution of fish in the beam, and measured SV becomes too small due to the border effect. Furusawa (2011b) [9] defines the school section scattering strength, that is the cumulation of all raw SV values in a school section, and the total school scattering strength, and then examines their characteristics using an exact computer simulation to show the effectiveness of the indexes.

Tang et al. (2003) [28] propose the EI of raw SV data obtained by a fisheries scanning sonar for surveying surface fish schools. The raw SV data in crescent-shape areas produced as a ship moves are integrated to give the average SV. Also, a new method termed the multibeam EI is devised for individual school abundance measurements $[9,20]$.

In the following, we examine the standard-sphere calibration method from the viewpoint of the above discussion. In particular, the standard-sphere echo integration, which has become one of the standard calibration methods, is discussed. As shown in Fig.10, the main calibration objects are the transmitreceive (TR) factor, Eq. (23), and the multiple echo factor, Eq. (25). The equivalent beam angle $\Psi$ included in the latter is calibrated by, for example, moving the sphere within the beam.

The TR factor can be determined by the average amplitude at a nearly flat part of the sphere echo using Eq. (22) (Fig.3b), using the TS value calculated by the theory. Since the definition of the TS in terms of the scattering theory is the squared scattering amplitude, it should be measured at a steady state part of the sphere echo not including transient parts. An EI over the whole waveform has introduced in the expression of backscattering cross section (an equivalent of the TS; see Table A) in [13], but the definition differs from the abovementioned definition. Also, in TS measurements of fish, even for partly overlapped echoes as shown in Fig.3c, we measure its TS at the nearly flat parts of the echoes after the single echo extraction processing (Fig.10) involving the criteria of amplitude and phase variations. If we measured TS's for whole echo shapes, the number of extracted echoes should be diminished.
In the past we obtained the equivalent pulse width $\tau$ by the numerical integration following Eq. (13) using a waveform observed at receiver output, but now we use the EI result of standard-sphere echoes. Such a method was introduced in [12] for a highly digitized echosounder, and the method was theoretically and experimentally confirmed in [23]. "SA of standard sphere" is obtained by substituting $S_{V}$ of Eq. (24), but with replacement of $E_{M}^{2}$ by $E_{S}^{2}$ of Eq. (22), into Eq. (17), and also including the wave function and the range correction or delay of Eq. (12):

$$
\begin{aligned}
S_{A s} & =\int_{r_{1}}^{r_{2}} E_{S}^{2} w^{2}\left(t-\frac{2 r_{s}}{c}\right) \cdot \frac{(r-\delta r)^{2} 10^{0.2 \alpha r}}{K_{M}^{\prime 2}} d r \\
& =\int_{r_{1}}^{r_{2}} K^{\prime 2} \frac{D^{4} T_{S}}{r_{s}^{4} 10^{0.2 \alpha r_{s}}} w^{2}\left(t-\frac{2 r_{s}}{c}\right) \cdot \frac{(r-\delta r)^{2} 10^{0.2 \alpha r}}{K_{M}^{\prime 2}} d r \\
& \equiv S_{A s 0} \frac{K^{\prime 2} \tau_{t}}{K^{\prime 2} \tau^{\prime}} \\
S_{A s 0} & =\frac{D^{4} T_{S}}{r_{s}^{2} \Psi} \\
\tau_{t} & =\int_{t_{s}}^{t_{s}+\tau_{m}} \frac{(t-2 \delta r / c)^{2}}{t_{s}^{2}} \cdot w^{2}\left(t-t_{s}\right) d t
\end{aligned}
$$

where, $r_{s}=c t_{s} / 2$ is the sphere range (see Fig.11), the absorption attenuation factors are ignored because of the smallness, and the primes indicate the old values used in the calibration exercise.

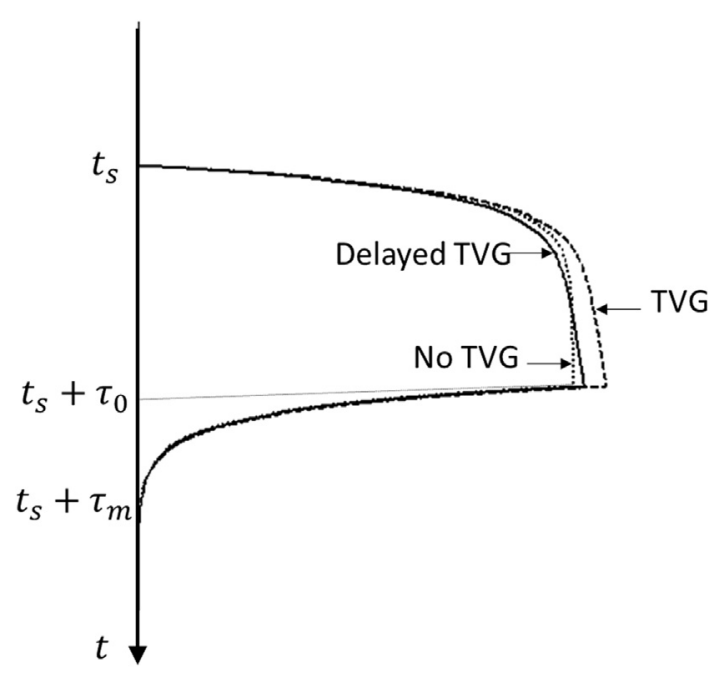

Fig. 11. Sphere echo shape with TVG effects. 


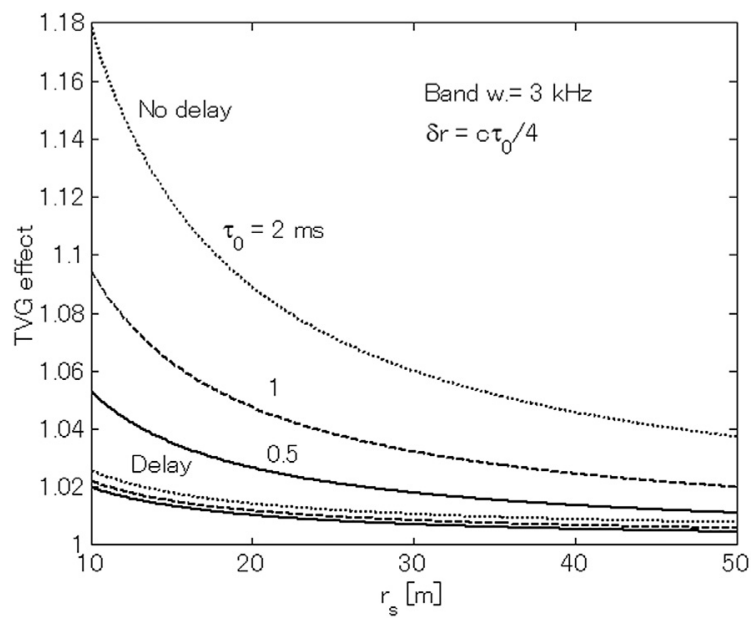

Fig. 12. TVG effect in sphere-echo integration. TVG with an appropriate delay makes the effect negligible.

Note that since we observe the echo shape after the $20 \log r$ TVG, the wave function is a little bit modified by the TVG [called TVG effect; the first factor in the integral of Eq. (28); see Fig.11] and the effect can be minimized by introducing the delay $\delta r$ shown in Eq. (12), especially for short ranges and large pulse widths. Fig. 12 shows the TVG effect, that is defined by $\tau_{t} / \tau$, computed for the wave function shown in Fig.11 [7,23]; this wave function is the response of a band limited system with a band width $\Delta f$ to a rectangular burst wave with a pulse width $\tau_{0}$. As seen in Fig.12, the TVG effect can be made as small as $1-2 \%$. The delay has been originally introduced to lessen the near range error in SV measurements $[7,14]$.

From Eq. (26), replacing $\tau_{t}$ by $\tau$, we finally have

$K^{2} \tau=K^{\prime 2} \tau^{\prime} \frac{S_{A s}}{S_{A s 0}}$

Since we calibrate the TR-factor $K$ by the separate sphere TS measurement, alternative calibration parameters may be given:

$\tau=\frac{K^{\prime 2}}{K^{2}} \tau^{\prime} \frac{S_{A s}}{S_{A s 0}}$ or $\frac{\tau}{\tau^{\prime}}=\frac{K^{\prime 2}}{K^{2}} \frac{S_{A s}}{S_{A s 0}}$

The sphere echo integration is performed in the multiple echo channel (the lower channel of Fig.10) for the single echo (sphere echo) using the EI function, that is the integration of single echo. This processing is, however, just a variant of the EI and only for the convenience of the calibration.

\section{Conclusions}

1) The basis of the echo integration (EI) method is the volume scattering theory (VST).
2) The VST holds for randomly and homogeneously distributed many fish school size of which is larger than a beam spread.

3) A measured volume backscattering strength (SV) according to VST is not always the intrinsic SV and should be called the raw SV.

4) The EI extends the applicability of the VST to various distributions and gives more precise results by introducing averaging, and the result is called the average SV.

5) The most primitive average $\mathrm{SV}$ is the ping average SV. The range averaging is not requisite, but it gives more stable average SV due to the ergodicity.

6) The area scattering strength (SA) is one of the EI and gives stable and useful measure proportional to the area density of fish.

7) Two separate calibrations of the transmit-receive factor $K$ and effective pulse width $\tau$ are necessary.

8) The standard-sphere EI effectively calibrates $K^{2} \tau$.

\section{Acknowledgment}

The author appreciates Mr. A. Okunishi (Furuno Electric Co.) for useful discussion. Two anonymous referees are thanked for their constructive comments.

\section{Appendix I. Terminogy and symbols}

Table A compares our terminology and symbols for scattering indexes with those recommended by ICES [17]. The explanations are in $[9,10]$.

\section{Appendix II. Normalized variance of echo integration result}

Moose and Ehrenberg (1971) [19] derived the normalized variance (square of coefficient of variation) of echo integration outputs on the basis of the inhomogeneous filtered-Poisson-process model as shown by [Eq. (21) of their paper].

$\frac{V A R_{I}}{E_{I}^{2}}=\frac{\mathrm{E}\left\{F^{4}\right\}}{\left(\mathrm{E}\left\{F^{2}\right\}\right)^{2} \lambda V\left(t_{1}, t_{2}\right)}+\frac{6 T_{p}\left(t_{2}^{5}-t_{1}^{5}\right)}{5\left(t_{2}^{3}-t_{1}^{3}\right)^{2}}$

where $V A R_{I}$ is the variance, $E_{I}$ is the average, $F$ is the single echo amplitude after TVG correction, $E\{\}$ stands for averaging, $\lambda$ is the average distribution density, $T_{p}$ is the pulse width, $t_{1}, t_{2}$ are integration interval, and $V\left(t_{1}, t_{2}\right)$ is the volume shown by 
$\mathrm{V}\left(t_{1}, t_{2}\right)=\frac{\pi}{24} c^{3} \sin \frac{\theta_{1}}{2} \sin \frac{\theta_{2}}{2}\left(t_{2}^{3}-t_{1}^{3}\right)$

where $c$ is the sound speed, and $\theta_{1}$ and $\theta_{2}$ are the beam widths in the alongship and athwartship directions, respectively.

Inserting the symbols which we use in the text, $T_{p}=\tau, \lambda_{0}=n, t=2 r / c$, and the following approximation

$\Psi=\pi \sin \frac{\theta_{1}}{2} \sin \frac{\theta_{2}}{2}$,

we have

$\frac{V A R_{I}}{E_{I}^{2}}=\frac{\mathrm{E}\left\{F^{4}\right\}}{\left(\mathrm{E}\left\{F^{2}\right\}\right)^{2}} \frac{3}{n \Psi\left(r_{2}^{3}-r_{1}^{3}\right)}+\frac{3 c \tau\left(r_{2}^{5}-r_{1}^{5}\right)}{5\left(r_{2}^{3}-r_{1}^{3}\right)^{2}}$

The $\left(\sigma_{I} / \mu_{I}\right)^{2}$ of Eq. (20) is obtained by dividing the above by the ping number according to Eq. (25) of [19]. In the reference further analysis is made for the case of overlapping beams and a further complicated expression is given, but the fundamental characteristics are expressed by the above nonoverlapping case.

The second term of the above equation shows the minimum asymptotic value when the VST holds, so that we examine the value when $r_{2}-r_{1}=c \tau / 2 \equiv r_{r}$ :

$$
\frac{3 c \tau\left(r_{2}^{5}-r_{1}^{5}\right)}{5\left(r_{2}^{3}-r_{1}^{3}\right)^{2}}=\frac{3 c \tau\left(1-\left[\frac{r_{2}-r_{r}}{r_{2}}\right]^{5}\right)}{5 r_{2}\left(1-\left[\frac{r_{2}-r_{r}}{r_{2}}\right]^{3}\right)^{2}} \cong \frac{3 c \tau\left(5 \frac{r_{r}}{r_{2}}\right)}{5 r_{2}\left(3 \frac{r_{r}}{r_{2}}\right)^{2}}=\frac{15 c \tau r_{r}}{45 r_{r}^{2}}=\frac{2}{3} .
$$

If we assume that the transition from the single echo scattering region, where the first term of Eq. (A1) is prevailing, to the volume scattering region, where the second term is prevailing, occurs at the point where the first term is $\kappa$ times the second term, then we have

$$
\frac{E\left\{F^{4}\right\}}{\left(E\left\{F^{2}\right\}\right)^{2}} \frac{1}{n \Psi}=\kappa \frac{c \tau\left(r_{2}^{5}-r_{1}^{5}\right)}{5\left(r_{2}^{3}-r_{1}^{3}\right)} \cong \kappa \frac{c \tau r_{2}^{5}\left(5 \frac{r_{r}}{r_{2}}\right)}{5 r_{2}^{3}\left(3 \frac{r_{r}}{r_{2}}\right)}=\kappa \frac{c \tau r_{2}^{2}}{3}
$$

and the transition range is

$r_{2}^{2}=\frac{3}{\kappa n \Psi_{C \tau}} \frac{E\left\{F^{4}\right\}}{\left(E\left\{F^{2}\right\}\right)^{2}}$.

The number of fish in the scattering volume at the transition range then becomes

$n \Psi r_{2}^{2} \frac{c \tau}{2}=\frac{1}{\kappa} \frac{3}{2} \frac{E\left\{F^{4}\right\}}{\left(E\left\{F^{2}\right\}\right)^{2}} \cong \frac{3}{\kappa}$

where the moment ratio $E\left\{F^{4}\right\} /\left(E\left\{F^{2}\right\}\right)^{2}$ is assumed to be 2 .

\begin{tabular}{|c|c|c|c|c|c|c|}
\hline \multicolumn{4}{|l|}{ This paper } & \multicolumn{3}{|l|}{ ICES recommendation } \\
\hline Name & Abbreviation & Definition & Unit & Name & Definition & Unit \\
\hline Target strength & TS & $\begin{array}{l}T_{S}=I_{r} / I_{i} \\
T S=10 \log T_{S}\end{array}$ & & $\begin{array}{l}\text { Backscattering cross section } \\
\text { Target strength }\end{array}$ & $\begin{array}{l}\sigma_{b s}=r_{0}^{2} L_{r} / L_{i} \\
T S=10 \log \sigma_{b s}\end{array}$ & $\begin{array}{l}\mathrm{m}^{2} \\
\mathrm{~dB}\end{array}$ \\
\hline $\begin{array}{l}\text { Volume backscattering } \\
\text { strength }\end{array}$ & SV & $\begin{array}{l}S_{V}=n T_{S} \\
S V=10 \log S_{V}\end{array}$ & $\begin{array}{l}1 / \mathrm{m}^{3} \\
\mathrm{~dB}\end{array}$ & $\begin{array}{l}\text { Volume backscattering coefficient } \\
\text { Volume backscattering strength }\end{array}$ & $\begin{array}{l}s_{v}=\sum \sigma_{b s / V} \\
S_{V}=10 \log s_{v}\end{array}$ & $\begin{array}{l}1 / \mathrm{m} \\
\mathrm{dB}\end{array}$ \\
\hline $\begin{array}{l}\text { Area backscattering } \\
\text { strength }\end{array}$ & SA & $\begin{array}{l}S_{A}=\int S_{V} d r \\
S A=10 \log S_{A}\end{array}$ & $\begin{array}{l}1 / \mathrm{m}^{2} \\
\mathrm{~dB}\end{array}$ & $\begin{array}{l}\text { Area backscattering coefficient } \\
\text { Area backscattering strength } \\
\text { Nautical area backscattering coefficient } \\
\text { Nautical area backscattering strength }\end{array}$ & $\begin{array}{l}s_{a}=\int s_{v} d r \\
s_{a}=10 \log s_{a} \\
s_{A}=4 \pi 1852^{2} s_{a} \\
S_{A}=10 \log s_{A}\end{array}$ & $\begin{array}{l}- \\
\mathrm{dB} \\
\mathrm{m}^{2} / \mathrm{nmi}^{2} \\
\mathrm{~dB}\end{array}$ \\
\hline
\end{tabular}

Table A. Terminology and symbols of scattering indexes adopted in this paper [10] and recommended by ICES [17].

$I_{i}$ : Incident intensity $\left[\mathrm{W} / \mathrm{m}^{2}\right], I_{r}:$ Scattered intensity at $\operatorname{lm}\left[\mathrm{W} / \mathrm{m}^{2}\right], r$ : Range $[\mathrm{m}], r_{0}$ : Reference range $(1 \mathrm{~m}), n$ : Distribution density $\left[1 / \mathrm{m}^{3}\right]$, $V$ : Volume occupied by scatterer $\left[\mathrm{m}^{3}\right]$. 


\section{References}

[1] Burczynski J. Introduction to the use of sonar systems for estimating fish biomass. Rome: FAO; 1982. FAO Fisheries Technical Paper, No.191, Revision 1.

[2] Demer D, Berger AL, Bernasconi M, Bethke E, Bosell K, Chu $\mathrm{D}$, et al. Calibration of acoustic instruments. Kopenhagen: ICES Cooperative Research Report; 2015. No.326.

[3] Dragesund O, Olsen S. On the possibility of estimating yearclass strength by measuring echo abundance of 0 -group fish. FiskDir Skr Havunder 1965;13:448-75.

[4] Eyring CF, Christensen RJ, Raitt RW. Reverberation in the sea. J Acoustical Soc Am 1948;20:462-75.

[5] Forbes ST, Nakken O, editors. Manual of methods for fisheries resource survey and appraisal. Part 2. The use of acoustic instruments for fish detection and abundance estimation. Rome: FAO Manuals in Fisheries Science. FAO; 1972.

[6] Furusawa M, Suzuki H, Miyanohana Y. Multi-purpose quantitative echo sounding system. J Marine Acoust Soc Japan 1989;16:82-93 [in Japanese, with English Abstract].

[7] Furusawa M, Hamada M, Aoyama C. Near range error in sound scattering measurements of fish. Fish Sci 1999;65: 108-15.

[8] Furusawa M. Echo integration near the seabed. J Marine Sci Technol 2011;19:259-66.

[9] Furusawa M. Section and total scattering strengths of individual fish school. J Marine Acoust Soc Japan 2011;38:177-94.

[10] Furusawa M, Amakasu K. Proposal to use fish-length-towavelength ratio characteristics of backscatter from fish for species identification. J Marine Acoust Soc Japan 2018;45: 183-96.

[11] Johannesson KA, Mitson RB. Fisheries acoustics - A practical manual for aquatic biomass estimation. Rome: FAO; 1983. FAO Fisheries Technical Paper 240.

[12] Knudsen HP. Bergen echo integrator: an introduction. J du Conseil Inter pour 1'Explorat de la Mer 1990;47:167-74.

[13] Lunde P, Korneliussen RJ. Power-budget equations and calibration factors for fish abundance estimation using scientific echo sounder and sonar systems. J Marine Sci Eng 2016;4:43. doi: 10.3390 .

[14] MacLennan DN. Time-varied-gain functions for pulsed sonars. J Sound Vibration 1986;110:511-22.

[15] MacLennan DN. Acoustical measurement of fish abundance. J Acoust Soc Am 1990;87:1-15.

[16] MacLennan DN, Simmonds EJ. Fisheries acoustics. London: Chapman and Hall; 1992.
[17] MacLennan DN, Fernandes PG, Dalen J. A consistent approach to definitions and symbols in fisheries acoustics. ICES J Marine Sci 2002;59:365-9.

[18] Manik HM, Furusawa M, Amakasu K. Measurement of sea bottom surface backscattering strength by quantitative echo sounder. Fish Sci 2006;72:503-12.

[19] Moose PH, Ehrenberg JE. An expression for the variance of abundance estimates using a fish echo integrator. J Fish Res Board Canada 1971;28:1293-301.

[20] Nishimori Y, Iida K, Furusawa M, Tang Y, Tokuyama K, Nagai S, Nishiyama Y. The development and evaluation of a three-dimensional, echo-integration method for estimating fish-school abundance. ICES J Marine Sci 2009;66:1037-42.

[21] Papoulis A. Probability, random variables, and stochastic processes. Tokyo: McGraw-Hill Kogakusha; 1965.

[22] Saneyoshi J, Nakamura K. Theory and model experiment on reflection of ultrasonic waves from numerous reflectors. J Acoust Soc Japan 1952;8:123-7 [in Japanese, with English Abstract].

[23] Sawada K, Furusawa M. Precision calibration of echo sounder by integration of sphere echoes. J Acoust Soc Japan (E) 1993;14:243-9.

[24] Simmonds EJ, MacLennan DN. Fisheries acoustics: theory and practice. 2nd ed. Oxford: Blackwell; 2005.

[25] Simrad. Simrad EK 500 operational manual (Theory of operation, 2 power budget). Horten, Norway. 1993.

[26] Smith PE. The horizontal dimensions and abundance of fish schools in the upper mixed layer as measured by sonar. In: Farquhar GB, editor. Proceedings of an international symposium on biological sound scattering in the ocean; $1970 . p$ 563-600. Virginia.

[27] Takao Y, Furusawa M. Noise measurement by echo integrator. Fish Sci 1995;61:637-40.

[28] Tang Y, Furusawa M, Aoyama S, Fan C, Nishimori Y. Measuring volume backscattering strength of surface fish schools by scanning sonar. Nippon Suisan Gakkaishi 2003, 69:153-61 [in Japanese, with English Abstract and Figure and Table captions].

[29] Thorne RE. Investigation into the relation between integrated echo voltage and fish density. J Fish Res Board Canada 1971;28:1269-73.

[30] Truskanov MD, Scherbino MN. Methods of direct calculation of fish concentrations by means of hydroacoustic apparatus. In: Paper presented to the seminar on fishery biology and Oceanography, Moscow; 1964.

[31] Urick RJ. Principle of underwater sound. Los Altos Hills, California: Peninsula Publishing; 1983. 